\title{
On the Role of Diffusion Behaviors in Stability Criterion for p-Laplace Dynamical Equations with Infinite Delay and Partial Fuzzy Parameters under Dirichlet Boundary Value
}

\author{
Ruofeng Rao,' ${ }^{1}$ Zhilin Pu, ${ }^{1,2}$ Shouming Zhong, ${ }^{1,3}$ and Jialin Huang ${ }^{1}$ \\ ${ }^{1}$ Institution of Mathematics, Yibin University, Yibin, Sichuan 644007, China \\ ${ }^{2}$ College of Mathematics and Software Science, Sichuan Normal University, Chengdu, Sichuan 610066, China \\ ${ }^{3}$ School of Science Mathematics, University of Electronic Science and Technology of China, Chengdu 610054, China \\ Correspondence should be addressed to Jialin Huang; jialinh28880@163.com
}

Received 23 July 2013; Revised 8 October 2013; Accepted 9 October 2013

Academic Editor: Reinaldo Martinez Palhares

Copyright ( 2013 Ruofeng Rao et al. This is an open access article distributed under the Creative Commons Attribution License, which permits unrestricted use, distribution, and reproduction in any medium, provided the original work is properly cited.

\begin{abstract}
By the way of Lyapunov-Krasovskii functional approach and some variational methods in the Sobolev space $W_{0}^{1, p}(\Omega)$, a global asymptotical stability criterion for $p$-Laplace partial differential equations with partial fuzzy parameters is derived under Dirichlet boundary condition, which gives a positive answer to an open problem proposed in some related literatures. Different from many previous related literatures, the nonlinear $p$-Laplace diffusion item plays its role in the new criterion though the nonlinear $p$-Laplace presents great difficulties. Moreover, numerical examples illustrate that our new stability criterion can judge what the previous criteria cannot do.
\end{abstract}

\section{Introduction}

Very recently, time-delay $p$-Laplace $(p>1)$ dynamical equations have attracted rapidly growing interest, because the nonlinear Laplace diffusion dynamical equations admit many physics and engineering background [1-6], such as CohenGrossberg neural networks and recurrent neural networks. In real world, diffusion phenomena cannot be unavoidable. Particularly, 2-Laplace $(p=2)$ is called the linear Laplace, and the diffusion phenomenon is always simulated by linear Laplace diffusion for simplicity ([7-10] and their references therein). However, diffusion behavior is so complicated that the nonlinear reaction-diffusion models were considered in many other recent literatures [1-6, 11-13]. Even the nonlinear $p$-Laplace diffusion $(p>1)$ is considered in simulating some diffusion behaviors [1-6]. But the previous related literature lost sight of the role of the nonlinear diffusion in their stability criteria. As pointed out in [2], the problem how the $p$-Laplace diffusion item plays a role in stability criteria remains open and challenging. And such a situation motivates our present study. Besides, fuzzy logic theory has been shown to be an appealing and efficient approach to dealing with the analysis and synthesis problems for complex nonlinear systems. Among various kinds of fuzzy methods, Takagi-Sugeno (TS) fuzzy models provide a successful method to describe certain complex nonlinear systems using some local linear subsystems [14-16]. Motivated by some ideas and methods of [17-21], we obtain a global asymptotical stability criterion for fuzzy T-S $p$-Laplace partial differential equations with Dirichlet boundary value by the way of Lyapunov-Krasovskii functional approach and some variational methods in the Sobolev space $W_{0}^{1, p}(\Omega)$. And in the obtained criterion, the nonlinear $p$-Laplace diffusion item plays a positive role.

\section{Model Description and Preliminaries}

Let us consider a class of fuzzy Takagi-Sugeno (T-S) $p$ Laplace partial differential equations described as follows. 
Fuzzy Rule $j$.

IF $\omega_{1}(t)$ is $\mu_{j 1}$ and $\cdots \omega_{s}(t)$ is $\mu_{j s}$, THEN

$$
\begin{aligned}
& \frac{\partial v}{\partial t}= \nabla \cdot\left(\Gamma(t, x, v) \circ \nabla_{p} v\right)-A(v) \\
& \times\left\{B(v)-\left[C_{j} f(v)+D_{j} g(v(t-\tau(t), x))\right]\right\}, \\
& v(\theta, x)=\phi(\theta, x), \quad(\theta, x) \in(-\infty, 0] \times \Omega, \\
& v(t, x)=0 \in R^{n}, \quad(t, x) \in R \times \partial \Omega,
\end{aligned}
$$

where $\Omega$ is an arbitrary open bounded subset in $R^{n}$. $\omega_{k}(t)(k=1,2, \ldots, s)$ is the premise variable, $\mu_{j k}(j=$ $1,2, \ldots, r ; k=1,2, \ldots, s)$ is the fuzzy set that is characterized by membership function. And $r$ is the number of the IF-THEN rules; $s$ is the number of the premise variables. Fuzzy partial differential system (1) admits its many physics and engineering background, including the famous Cohen-Grossberg neural networks [1-6]. Consider $v(t, x)=$ $\left(v_{1}(t, x), v_{2}(t, x), \ldots, v_{n}(t, x)\right)^{T} \in R^{n}$, where $v_{i}(t, x)$ is the state variable of the $i$ th neuron and the $j$ th neuron at time $t$ and in space variable $x$. Matrix $\Gamma(t, x, v)=\left(\Gamma_{i j}(t, x, v)\right)_{n \times m}$ with each $\Gamma_{i j}(t, x, v) \geqslant 0$, and $\Gamma_{i j}(t, x, v)$ is diffusion operator. $\Gamma(t, x, v) \circ \nabla_{p} v=\left(\Gamma_{i k}(t, x, v)\left|\nabla v_{i}\right|^{p-2}\left(\partial v_{i} / \partial x_{k}\right)\right)_{n \times m}$ denotes the Hadamard product of matrix $\Gamma(t, x, v)$ and $\nabla_{p} v$ (see [1$6]$ for details). Matrix $A(v)=\operatorname{diag}\left(a_{1}\left(v_{1}\right), a_{2}\left(v_{2}\right), \ldots, a_{n}\left(v_{n}\right)\right)$ and vector $B(v)=\left(b_{1}\left(v_{1}\right), b_{2}\left(v_{2}\right), \ldots, b_{n}\left(v_{n}\right)\right)^{T}$, where $a_{i}\left(v_{i}\right)$ and $b_{i}\left(v_{i}\right)$ represent an amplification function at time $t$ and an appropriately behaved function at time $t . C_{j}=$ $\left(c_{i k}^{(j)}\right)_{n \times n}$ and $D_{j}=\left(d_{i k}^{(j)}\right)_{n \times n}$ are connection matrices. The time-varying delays are $\tau(t) \in[0,+\infty) . f(v)=$ $\left(f_{1}\left(v_{1}\right), f_{2}\left(v_{2}\right), \ldots, f_{n}\left(v_{n}\right)\right)^{T}$ and $g(v(t-\tau(t)))=\left(g_{1}\left(v_{1}(t-\right.\right.$ $\left.\tau(t))), g_{2}\left(v_{2}(t-\tau(t))\right), \ldots, g_{n}\left(v_{n}(t-\tau(t))\right)\right)^{T}$ are the activation functions of the neurons. And the second and third equations of (1) represent the initial condition and the Dirichlet boundary condition, respectively.

With the help of a standard fuzzy inference method, (1) can be inferred as follows:

$$
\begin{gathered}
\frac{\partial v}{\partial t}=\nabla \cdot\left(\Gamma(t, x, v) \circ \nabla_{p} v\right) \\
-A(v)\left\{B(v)-\sum_{j=1}^{r} h_{j}(\omega(t))\right. \\
\times\left[C_{j} f(v)+D_{j} g\right. \\
\times(v(t-\tau(t), x))]\}, \\
v(\theta, x)=\phi(\theta, x), \quad(\theta, x) \in(-\infty, 0] \times \Omega, \\
v(t, x)=0 \in R^{n}, \quad(t, x) \in R \times \partial \Omega,
\end{gathered}
$$

where $\omega(t)=\left[\omega_{1}(t), \omega_{2}(t), \ldots, \omega_{s}(t)\right], h_{j}(\omega(t))=\left(w_{j}(\omega(t))\right) /$ $\left(\sum_{k=1}^{r} w_{k}(\omega(t))\right), w_{j}(\omega(t)): R^{s} \rightarrow[0,1](j=1,2, \ldots, r)$ is the membership function of the system with respect to the fuzzy rule $j . h_{j}$ can be regarded as the normalized weight of each IF-THEN rule, satisfying $h_{j}(\omega(t)) \geqslant 0$ and $\sum_{j=1}^{r} h_{j}(\omega(t))=1$.

For convenience's sake, we need to introduce some standard notations.

(i) Denote $|C|=\left(\left|c_{i j}\right|\right)_{n \times n}$ for any matrix $C=\left(c_{i j}\right)_{n \times n}$.

(ii) Denote $|v(t, x)|=\left(\left|v_{1}(t, x)\right|,\left|v_{2}(t, x)\right|, \ldots,\left|v_{n}(t, x)\right|\right)^{T}$ for any $v(t, x)=\left(v_{1}(t, x), v_{2}(t, x), \ldots, v_{n}(t, x)\right)^{T}$.

(iii) $u \geqslant v$ if $u_{i} \geqslant v_{i}$ for all $i=1,2, \ldots, n$, where $u=$ $\left(u_{1}, u_{2}, \ldots, u_{n}\right)^{T}, v=\left(v_{1}, v_{2}, \ldots, v_{n}\right)^{T} \in R^{n}$.

In addition, we also introduce the following standard notations similarly as $[2,(\mathrm{iii})-(\mathrm{X})]$ :

$$
\begin{gathered}
Q=\left(q_{i j}\right)_{n \times n}>0(<0), \quad Q=\left(q_{i j}\right)_{n \times n} \geqslant 0(\leqslant 0), \\
Q_{1} \geqslant Q_{2}\left(Q_{1} \leqslant Q_{2}\right),
\end{gathered}
$$

$Q_{1}>Q_{2}\left(Q_{1}<Q_{2}\right)$, and the identity matrix $I$.

Throughout this paper, we assume the following.

(H1) There exist positive definite matrices $\underline{A}=\operatorname{diag}\left(\underline{a}_{1}\right.$, $\left.\underline{a}_{2}, \ldots, \underline{a}_{n}\right)$ and $\bar{A}=\operatorname{diag}\left(\bar{a}_{1}, \bar{a}_{2}, \ldots, \bar{a}_{n}\right)$ such that

$$
0<\underline{a}_{i} \leqslant a_{i}(r) \leqslant \bar{a}_{i}, \quad r \in R, i=1,2, \ldots, n .
$$

(H2) There exists a positive definite matrix $B=$ $\operatorname{diag}\left(b_{1}, b_{2}, \ldots, b_{n}\right)$ such that $b_{i}(0)=0$ and

$$
\frac{b_{i}(r)}{r^{p-1}} \geqslant b_{i}, \quad 0 \neq r \in R, i=1,2, \ldots, n .
$$

(H3) There exist positive definite matrices $F=\operatorname{diag}\left(F_{1}\right.$, $\left.F_{2}, \ldots, F_{n}\right)$ and $G=\operatorname{diag}\left(G_{1}, G_{2}, \ldots, G_{n}\right)$ such that

$$
\begin{array}{r}
\left|f_{i}(r)\right| \leqslant F_{i}|r|^{p-1}, \quad\left|g_{i}(r)\right| \leqslant G_{i}|r|^{p-1}, \quad r \in R, \\
i=1,2, \ldots, n .
\end{array}
$$

From (H1)-(H3), we know that $f(0)=g(0)=0 \in R^{n}$, and $v=0$ is an equilibrium of (2).

Lemma 1. For any $a, b \in(0,+\infty)$, One has

$$
a^{p-1} b \leqslant \frac{p-1}{p} a^{p}+\frac{b^{p}}{p} .
$$

\section{Main Result}

Before giving the main result of this paper, we have to present the following Lemma via some variational methods in the Sobolev space $W_{0}^{1, p}(\Omega)$, which is the completion of $C_{0}^{\infty}(\Omega)$ with respect to the norm $\|\eta\|=\left(\int_{\Omega}|\nabla \eta(x)|^{p} d x\right)^{1 / p}$. Denote by $\lambda_{1}$ the first eigenvalue of $-\Delta_{p}$ in Sobolev space $W_{0}^{1, p}(\Omega)$, where $\lambda_{1}=\min \left\{\int_{\Omega}|\nabla \eta(x)|^{p} d x: \eta(x) \in W_{0}^{1, p}(\Omega)\right.$, $\left.\int_{\Omega}|\eta(x)|^{p} d x=1\right\}$ (see [22-24] for details). 
Lemma 2. Let $Q=\operatorname{diag}\left(q_{1}, q_{2}, \ldots, q_{n}\right)$ be a positive definite matrix, and let $v$ be a solution of the fuzzy system (2). Then One has

$$
\int_{\Omega} v^{T} Q\left(\nabla \cdot\left(\Gamma(t, x, v) \circ \nabla_{p} v\right)\right) d x \leqslant-\lambda_{1} \underline{q} \Gamma\|v\|_{p}^{p},
$$

where $\Gamma=\min _{i, k}\left(\inf _{t, x, v} \Gamma_{i k}(t, x, v)\right),\|v\|_{p}^{p}=\sum_{i=1}^{n} \int_{\Omega}\left|v_{i}\right|^{p} d x$, and $\underline{q}$ is a positive scalar, satisfying $Q>\underline{q} I$.

Proof. Since $v$ is a solution of system (2), it follows by Gauss formula and the Dirichlet boundary condition that

$$
\begin{gathered}
\int_{\Omega} v^{T} Q\left(\nabla \cdot\left(\Gamma(t, x, v) \circ \nabla_{p} v\right)\right) d x \\
=\int_{\Omega} v^{T} Q\left(\sum_{k=1}^{m} \frac{\partial}{\partial x_{k}}\left(\Gamma_{1 k}\left|\nabla v_{1}\right|^{p-2} \frac{\partial v_{1}}{\partial x_{k}}\right), \ldots,\right. \\
\left.\sum_{k=1}^{m} \frac{\partial}{\partial x_{k}}\left(\Gamma_{n k}\left|\nabla v_{n}\right|^{p-2} \frac{\partial v_{n}}{\partial x_{k}}\right)\right)^{T} d x \\
=-\sum_{k=1}^{m} \sum_{j=1}^{n} \int_{\Omega} q_{j} \Gamma_{j k}\left|\nabla v_{j}\right|^{p-2}\left(\frac{\partial v_{j}}{\partial x_{k}}\right)^{2} d x \\
\leqslant-\lambda_{1} \underline{q} \Gamma\|v\|_{p}^{p} .
\end{gathered}
$$

Remark 3. Lemma 2 actually generalizes the conclusion of [7, Lemma 2.1] and [25, Lemma 2.4] from Hilbert space $H_{0}^{1}(\Omega)$ to Banach space $W_{0}^{1, p}(\Omega)$. Particularly in the case of $\Omega=(0, T) \subset R^{1}$ or $W_{0}^{1, p}(0, T)$, the first eigenvalue $\lambda_{1}=$ $\left((2 / T) \int_{0}^{(p-1)^{1 / p}}\left(d t /\left(1-\left(t^{p} /(p-1)\right)\right)^{1 / p}\right)\right)^{p}$ (see, e.g., [22]).

Theorem 4. Suppose that $p=m_{1} / m_{2}>1$, where $m_{1}$ is an even number while $m_{2}$ is an odd number. If, in addition, there exist a positive definite matrix $Q=\operatorname{diag}\left(q_{1}, q_{2}, \ldots, q_{n}\right)$ and two positive scalars $q, \bar{q}$ such that the following inequalities hold:

$$
\begin{aligned}
& {\left[\lambda_{1} \Gamma+\lambda_{\min }(\underline{A} B)\right] \underline{q}} \\
& >\left[n\left(\lambda_{\max } \bar{A}\right)\left(\lambda_{\max } F\right)\right. \\
& \quad \times \sum_{j=1}^{r}\left|c_{j}\right|+\left(\frac{n}{p}+\frac{n(p-1)}{(1-\tau) p}\right) \\
& \left.\times\left(\lambda_{\max } \bar{A}\right)\left(\lambda_{\max } G\right) \sum_{j=1}^{r}\left|d_{j}\right|\right] \bar{q}, \\
& q_{i}>\underline{q}, \quad i \in \mathcal{N}, \\
& q_{i}<\bar{q}, \quad i \in \mathcal{N},
\end{aligned}
$$

then the null solution of (2) is globally asymptotically stable, where $\mathcal{N}=\{1,2, \ldots, n\}, \tau^{\prime}(t) \leqslant \tau<1,\left|c_{j}\right|=\max _{i, k}\left|c_{i k}^{(j)}\right|$ for the matrix $C_{j}=\left(c_{i k}^{(j)}\right)_{n \times n}$, and $\left|d_{j}\right|=\max _{i, k}\left|d_{i k}^{(j)}\right|$ for the matrix $D_{j}=\left(d_{i k}^{(j)}\right)_{n \times n}$.

Proof. Define the Lyapunov-Krasovskii functional as follows:

$$
V(t)=V_{1}(t)+\frac{1}{1-\tau} V_{2}(t)
$$

where

$$
\begin{gathered}
V_{1}(t)=\int_{\Omega} v^{T} Q v d x, \\
V_{2}(t)=2 n \bar{q}\left(\lambda_{\max } \bar{A}\right)\left(\lambda_{\max } G\right) \frac{p-1}{p} \\
\times \sum_{j=1}^{r} \sum_{k=1}^{n}\left|d_{j}\right| \int_{t-\tau(t)}^{t} \int_{\Omega}\left|v_{k}(s, x)\right|^{p} d s d x .
\end{gathered}
$$

Evaluating the time derivation of $V_{1}(t)$ along the trajectory of the fuzzy system (2), we can get by Lemma 2

$$
\begin{aligned}
V_{1}^{\prime}(t) \leqslant & -2 \lambda_{1} \underline{q} \Gamma\|v\|_{p}^{p} \\
& -2 \int_{\Omega} v^{T} Q A(v) B(v) d x \\
& +2 \sum_{j=1}^{r} h_{j}(\omega(t)) \int_{\Omega} v^{T} Q\left[A(v) C_{j} f(v)\right. \\
& \left.+A(v) D_{j} g\left(v_{\tau}\right)\right] d x \\
\leqslant & -2 \lambda_{1} \underline{q} \Gamma\|v\|_{p}^{p}-2 \int_{\Omega} v^{T} Q A(v) B(v) d x \\
& +2 \sum_{j=1}^{r} \int_{\Omega}\left|v^{T}\right| Q\left[\bar{A}\left|C_{j}\right||f(v)|+\bar{A}\left|D_{j}\right|\left|g\left(v_{\tau}\right)\right|\right] d x,
\end{aligned}
$$

where we denote $v_{\tau}=v(t-\tau(t), x)$ for convenience.

Then we can get by (H1), (H2) and the restrictive conditions on the parameter $p$

$$
\begin{aligned}
\int_{\Omega} v^{T} Q A(v) B(v) d x \\
\quad \geqslant \underline{q} \int_{\Omega}\left|v^{T}\right| \underline{A} B\left(\left|v_{1}\right|^{p-1},\left|v_{2}\right|^{p-1}, \ldots,\left|v_{n}\right|^{p-1}\right)^{T} d x \\
\quad \geqslant \underline{q} \lambda_{\min }(\underline{A} B)\|v\|_{p}^{p} .
\end{aligned}
$$

It follows by (H3) and Lemma 1 that

$$
\begin{aligned}
& \int_{\Omega}\left|v^{T}\right| Q \bar{A}\left|C_{j}\right||f(v)| d x \\
& \quad \leqslant \int_{\Omega}\left|v^{T} Q\right| \bar{A}\left|C_{j}\right| F\left(\left|v_{1}\right|^{p-1},\left|v_{2}\right|^{p-1}, \ldots,\left|v_{n}\right|^{p-1}\right)^{T} d x \\
& \quad=\bar{q} \sum_{k=1}^{n} \sum_{i=1}^{n} \int_{\Omega}\left|v_{i}\right| \bar{a}_{i}\left|c_{i k}^{(j)}\right| F_{k}\left|v_{k}\right|^{p-1} d x
\end{aligned}
$$




$$
\begin{aligned}
\leqslant & \bar{q} \sum_{k=1}^{n} \sum_{i=1}^{n} \int_{\Omega} \bar{a}_{i}\left|c_{i k}^{(j)}\right| F_{k}\left(\frac{p-1}{p}\left|v_{k}\right|^{p}+\frac{\left|v_{i}\right|^{p}}{p}\right) d x \\
= & \bar{q} \frac{p-1}{p} \sum_{k=1}^{n} \sum_{i=1}^{n} \int_{\Omega} \bar{a}_{i}\left|c_{i k}^{(j)}\right| F_{k}\left|v_{k}\right|^{p} d x \\
& +\bar{q} \frac{1}{p} \sum_{k=1}^{n} \sum_{i=1}^{n} \int_{\Omega} \bar{a}_{i}\left|c_{i k}^{(j)}\right| F_{k}\left|v_{i}\right|^{p} d x \\
\leqslant & \bar{q}\left|c_{j}\right|\left(\lambda_{\max } \bar{A}\right)\left(\lambda_{\max } F\right) \\
& \times\left(\frac{p-1}{p} \sum_{k=1 i=1}^{n} \sum_{\Omega}^{n}\left|v_{k}\right|^{p} d x+\frac{1}{p} \sum_{k=1 i=1}^{n} \sum_{\Omega}^{n} \int_{\Omega}\left|v_{i}\right|^{p} d x\right) \\
= & n \bar{q}\left|c_{j}\right|\left(\lambda_{\max } \bar{A}\right)\left(\lambda_{\max } F\right) \\
& \times\left(\frac{p-1}{p} \sum_{k=1}^{n} \int_{\Omega}\left|v_{k}\right|^{p} d x+\frac{1}{p} \sum_{i=1}^{n} \int_{\Omega}\left|v_{i}\right|^{p} d x\right) \\
= & n \bar{q}\left|c_{j}\right|\left(\lambda_{\max } \bar{A}\right)\left(\lambda_{\max } F\right)\|v\|_{p}^{p} .
\end{aligned}
$$

Similarly,

$$
\begin{aligned}
& \int_{\Omega}\left|v^{T}\right| Q \bar{A}\left|D_{j}\right|\left|g\left(v_{\tau}\right)\right| d x \\
& \leqslant \bar{q} \sum_{k=1}^{n} \sum_{i=1}^{n} \int_{\Omega} \bar{a}_{i}\left|d_{i k}^{(j)}\right| G_{k} \\
& \times\left(\frac{p-1}{p}\left|v_{k}(t-\tau(t), x)\right|^{p}+\frac{\left|v_{i}(t, x)\right|^{p}}{p}\right) d x \\
& \leqslant n \bar{q}\left|d_{j}\right|\left(\lambda_{\max } \bar{A}\right)\left(\lambda_{\max } G\right) \\
& \times\left(\frac{p-1}{p} \sum_{k=1}^{n} \int_{\Omega}\left|v_{k}(t-\tau(t), x)\right|^{p} d x+\frac{1}{p}\|v\|_{p}^{p}\right) .
\end{aligned}
$$

Combining (15)-(18) results in

$$
\begin{aligned}
V_{1}^{\prime}(t) \leqslant & -2\left(\lambda_{1} \underline{q}_{\underline{q}}+\underline{q} \lambda_{\min }(\underline{A} B)-n \bar{q}\left(\lambda_{\max } \bar{A}\right)\left(\lambda_{\max } F\right)\right. \\
& \left.\times \sum_{j=1}^{r}\left|c_{j}\right|-\frac{1}{p} n \bar{q}\left(\lambda_{\max } \bar{A}\right)\left(\lambda_{\max } G\right) \sum_{j=1}^{r}\left|d_{j}\right|\right) \\
& \times\|v\|_{p}^{p}+2 n \bar{q}\left(\lambda_{\max } \bar{A}\right)\left(\lambda_{\max } G\right) \frac{p-1}{p} \\
& \times \sum_{j=1}^{r} \sum_{k=1}^{n}\left|d_{j}\right| \int_{\Omega}\left|v_{k}(t-\tau(t), x)\right|^{p} d x .
\end{aligned}
$$

On the other hand,

$$
\begin{aligned}
V_{2}^{\prime}(t)= & 2 n \bar{q}\left(\lambda_{\max } \bar{A}\right)\left(\lambda_{\max } G\right) \frac{p-1}{p}\|v\|_{p}^{p} \sum_{j=1}^{r}\left|d_{j}\right| \\
& -\left(1-\tau^{\prime}(t)\right) 2 n \bar{q}\left(\lambda_{\max } \bar{A}\right)\left(\lambda_{\max } G\right) \frac{p-1}{p} \\
& \times \sum_{j=1}^{r} \sum_{k=1}^{n}\left|d_{j}\right| \int_{\Omega}\left|v_{k}(t-\tau(t), x)\right|^{p} d x \\
\leqslant & 2 n \bar{q}\left|d_{j}\right|\left(\lambda_{\max } \bar{A}\right)\left(\lambda_{\max } G\right) \frac{p-1}{p}\|v\|_{p}^{p} \sum_{j=1}^{r}\left|d_{j}\right| \\
& -(1-\tau) 2 n \bar{q}\left(\lambda_{\max } \bar{A}\right)\left(\lambda_{\max } G\right) \frac{p-1}{p} \\
& \times \sum_{j=1}^{r} \sum_{k=1}^{n}\left|d_{j}\right| \int_{\Omega}\left|v_{k}(t-\tau(t), x)\right|^{p} d x .
\end{aligned}
$$

So we conclude from (10) that

$$
\begin{aligned}
V^{\prime}(t) \leqslant-2\left(\lambda_{1} \underline{q} \Gamma+\underline{q} \lambda_{\min }(\underline{A} B)-n \bar{q}\left(\lambda_{\max } \bar{A}\right)\left(\lambda_{\max } F\right)\right. \\
\quad \times \sum_{j=1}^{r}\left|c_{j}\right|-\frac{1}{p} n \bar{q}\left(\lambda_{\max } \bar{A}\right)\left(\lambda_{\max } G\right) \sum_{j=1}^{r}\left|d_{j}\right| \\
\left.\quad-\frac{1}{1-\tau} n \bar{q}\left(\lambda_{\max } \bar{A}\right)\left(\lambda_{\max } G\right) \frac{p-1}{p} \sum_{j=1}^{r}\left|d_{j}\right|\right) \\
\times\|v\|_{p}^{p} \leqslant 0 .
\end{aligned}
$$

It follows by the standard Lyapunov functional theory that the null solution of the fuzzy system (2) is globally asymptotically stable.

Remark 5. In many previous related literatures (see, e.g., [1$6]$ ), the nonlinear $p$-Laplace $(p>2)$ diffusion terms were omitted in the deductions, which results in that their stability criteria do not contain the diffusion terms. In other words, the diffusion terms do not affect their results. In addition, when $p=2,2$-Laplace is the linear Laplace, and there are many papers (see, e.g., [25-29]) in which the Laplace diffusion item plays its role in their stability criteria, for the linear Laplace PDEs can be considered in the special Hilbert space $H^{1}(\Omega)$ that can be orthogonally decomposed into the direct sum of infinitely many eigenfunction spaces. However, the nonlinear $p$-Laplace $(p>1, p \neq 2)$ brings great difficulties, for the nonlinear $p$-Laplace PDEs should be considered in the frame of Sobolev space $W^{1, p}(\Omega)$ that is only a reflexive Banach space. Indeed, owing to the great difficulties, the authors only provide in [4] the stability criterion in which the nonlinear $p$-Laplace items play roles in the case of $1<p<2$. However, in this paper, the nonlinear $p$-Laplace diffusion terms play a positive role in our Theorem 4 for the case of $p>2$ or 
$p>1$, which also gives a positive answer to the open problem proposed in [2] to some extent. Besides, we will provide a numerical example where our Theorem 4 works whereas [2, Corollary 15] do not (see, Example 2).

Remark 6. Particularly when $p=2$, Theorem 4 provides a global asymptotical stability criterion for the familiar reaction-diffusion fuzzy CGNNs with infinite delay. Even in this particular case, the result is also good thanks to the infinite allowable upper bounds of time delays.

\section{Numerical Example}

Example 1. Consider the $p$-Laplace fuzzy T-S dynamic equations as follows.

Fuzzy Rule 1.

$$
\begin{aligned}
& \text { IF } \omega_{1}(t) \text { is } 1 / e^{-2 \omega_{1}(t)}, \text { THEN } \\
& \begin{aligned}
\frac{\partial v}{\partial t}= & \nabla \cdot\left(\Gamma(t, x, v) \circ \nabla_{p} v\right) \\
& -A(v)\left\{B(v)-\left[C_{1} f(v)+D_{1} g(v(t-\tau(t), x))\right]\right\}, \\
v & (\theta, x)=\phi(\theta, x), \quad(\theta, x) \in(-\infty, 0] \times \Omega, \\
& v(t, x)=0 \in R^{2}, \quad(t, x) \in R \times \partial \Omega .
\end{aligned}
\end{aligned}
$$

Fuzzy Rule 2.

$$
\begin{aligned}
& \text { IF } \omega_{2}(t) \text { is } 1-\left(1 / e^{-2 \omega_{1}(t)}\right), \text { THEN } \\
& \begin{aligned}
\frac{\partial v}{\partial t}= & \nabla \cdot\left(\Gamma(t, x, v) \circ \nabla_{p} v\right)-A(v) \\
& \times\left\{B(v)-\left[C_{2} f(v)+D_{2} g(v(t-\tau(t), x))\right]\right\}, \\
v & (\theta, x)=\phi(\theta, x), \quad(\theta, x) \in(-\infty, 0] \times \Omega, \\
& v(t, x)=0 \in R^{2}, \quad(t, x) \in R \times \partial \Omega,
\end{aligned}
\end{aligned}
$$

where $v(t, x)=\left(v_{1}(t, x), v_{2}(t, x)\right)^{T}, \Omega=(0, \pi), p=4 / 3$, and then the first eigenvalue $\lambda_{1}=\left((2 / \pi) \int_{0}^{(p-1)^{1 / p}}\left(d t /\left(1-\left(t^{p} /(p-\right.\right.\right.\right.$ $\left.\left.1)))^{1 / p}\right)\right)^{p}=0.7915$ (see Remark 3$)$. The initial value function is presented as follows:

$$
\phi(s, x)=\left(\begin{array}{c}
0.25\left(1-\cos \left(5 \pi x^{2}\right)\right) \sin ^{398}\left(x^{2}-0.25\right) e^{-100 s} \\
0.2 \cos ^{2}\left(4 \pi x^{2}\right) \sin ^{508}\left(x^{2}-0.55\right) e^{-100 s}
\end{array}\right) .
$$

Let $\tau(t)=t / 3$, and then $\tau^{\prime}(t) \leqslant 1 / 3=\tau<1$. Besides, $A(v)=\operatorname{diag}\left(0.7+0.2 \sin ^{2}\left(v_{1}\right), 0.6+0.28 \sin ^{2}\left(v_{2}\right)\right), B(v)=$ $\left(2 v_{1}^{1 / 3}, 1.9 v_{2}^{1 / 3}\right)^{T}$, and $f(v)=g(v)=\left(0.1 v_{1}^{1 / 3}, 0.2 v_{2}^{1 / 3}\right)^{T}$, and correspondingly we assume

$$
\begin{array}{ll}
\underline{A}=\left(\begin{array}{cc}
0.7 & 0 \\
0 & 0.6
\end{array}\right), & \bar{A}=\left(\begin{array}{cc}
0.9 & 0 \\
0 & 0.88
\end{array}\right), \\
B=\left(\begin{array}{cc}
2 & 0 \\
0 & 1.9
\end{array}\right), & F=\left(\begin{array}{cc}
0.1 & 0 \\
0 & 0.2
\end{array}\right)=G .
\end{array}
$$

In addition, $r=2$, and

$$
\begin{gathered}
C_{1}=\left(\begin{array}{cc}
-0.2 & 0.3 \\
0.28 & 0.3
\end{array}\right)=D_{1}, \quad C_{2}=\left(\begin{array}{cc}
0.29 & 0.18 \\
0.2 & -0.1
\end{array}\right)=D_{2}, \\
\Gamma(t, x, v)=\left(\begin{array}{cc}
0.008 & 0.007 \\
0.006 & 0.006
\end{array}\right) .
\end{gathered}
$$

Hence, $\Gamma=0.006,\left|c_{1}\right|=\left|d_{1}\right|=0.3,\left|c_{2}\right|=\left|d_{2}\right|=0.29$, $\lambda_{\min }(\underline{A B})=1.1400, \lambda_{\max } \bar{A}=0.9$, and $\lambda_{\max } F=\lambda_{\max } G=$ 0.2 . Then we can use MATLAB LMI toolbox to solve the inequalities (10)-(12), and obtain $t_{\min }=-0.5569<0$ which implies feasible. Further, extracting the datum shows $\underline{q}=$ 19.7994, $\bar{q}=31.1620, q_{i}=24.3210, i=1,2$. Thereby, we can conclude from Theorem 4 that the null solution for this fuzzy dynamic equation is globally asymptotically stable (see Figures 1 and 2).

Example 2. To compare our Theorem 4 with [2, Corollary 15], we may consider the following $p$-Laplace dynamic equations.

$$
\begin{gathered}
\frac{\partial v}{\partial t}=\nabla \cdot\left(\Gamma(t, x, v) \circ \nabla_{3} v\right) \\
-A(v)\{B(v)-[C f(v)+D g(v(t-30, x))]\}, \\
v(\theta, x)=\phi(\theta, x), \quad(\theta, x) \in(-\infty, 0] \times \Omega, \\
v(t, x)=0 \in R^{2}, \quad(t, x) \in R \times \partial \Omega,
\end{gathered}
$$

where $v(t, x)=\left(v_{1}(t, x), v_{2}(t, x)\right)^{T}, \Omega=(0, \pi / 2), p=3$, and then the first eigenvalue $\lambda_{1}=7.3104$ (see Remark 5). Let $\tau(t) \equiv 30$, and then $\tau^{\prime}(t) \equiv 0<1$. In addition, $r=1$ is the number of the IF-THEN. Assume

$$
\begin{array}{cc}
\underline{A}=\left(\begin{array}{cc}
0.7 & 0 \\
0 & 0.6
\end{array}\right), & \bar{A}=\left(\begin{array}{cc}
0.9 & 0 \\
0 & 0.88
\end{array}\right), \\
B=\left(\begin{array}{cc}
0.2 & 0 \\
0 & 0.1
\end{array}\right), & F=\left(\begin{array}{cc}
0.1 & 0 \\
0 & 0.2
\end{array}\right)=G, \\
C=\left(\begin{array}{cc}
-0.2 & 0.3 \\
0.3 & 0.3
\end{array}\right)=D, \quad \Gamma(t, x, v)=\left(\begin{array}{cc}
0.08 & 0.07 \\
0.06 & 0.06
\end{array}\right) .
\end{array}
$$

Below we shall employ [2, Corollary 15] and our Theorem 4 to judge the stability of System (27), respectively.

In [2, Corollary 15], letting $\lambda=0.001$, we use MATLAB LMI toolbox to solve the LMI conditions [2, (37)] and obtain $t_{\min }=7.1357 \times 10^{-13}>0$, which implies that these LMI constraints are not strictly feasible. Again, let $\lambda=1$ and $\lambda=$ 10 in [2, Corollary 15], respectively. And then $t_{\min }=2.1479 \times$ $10^{-20}>0$ and $t_{\min }=2.1026 \times 10^{-16}>0$, respectively. Hence, we cannot judge the stability of System (27) from [2, Corollary 15]. However, the LMIs of [2, Corollary 15] are only the sufficient condition for the stability, not necessary for stability. Indeed, we use MATLAB LMI toolbox to solve (10)-(12) in Theorem 4 and obtain $t_{\min }=-0.0794<0$, which implies being feasible. Further, extracting the datum results in that $q=0.9803, \bar{q}=1.8381$, and $q_{i}=1.3671$, 

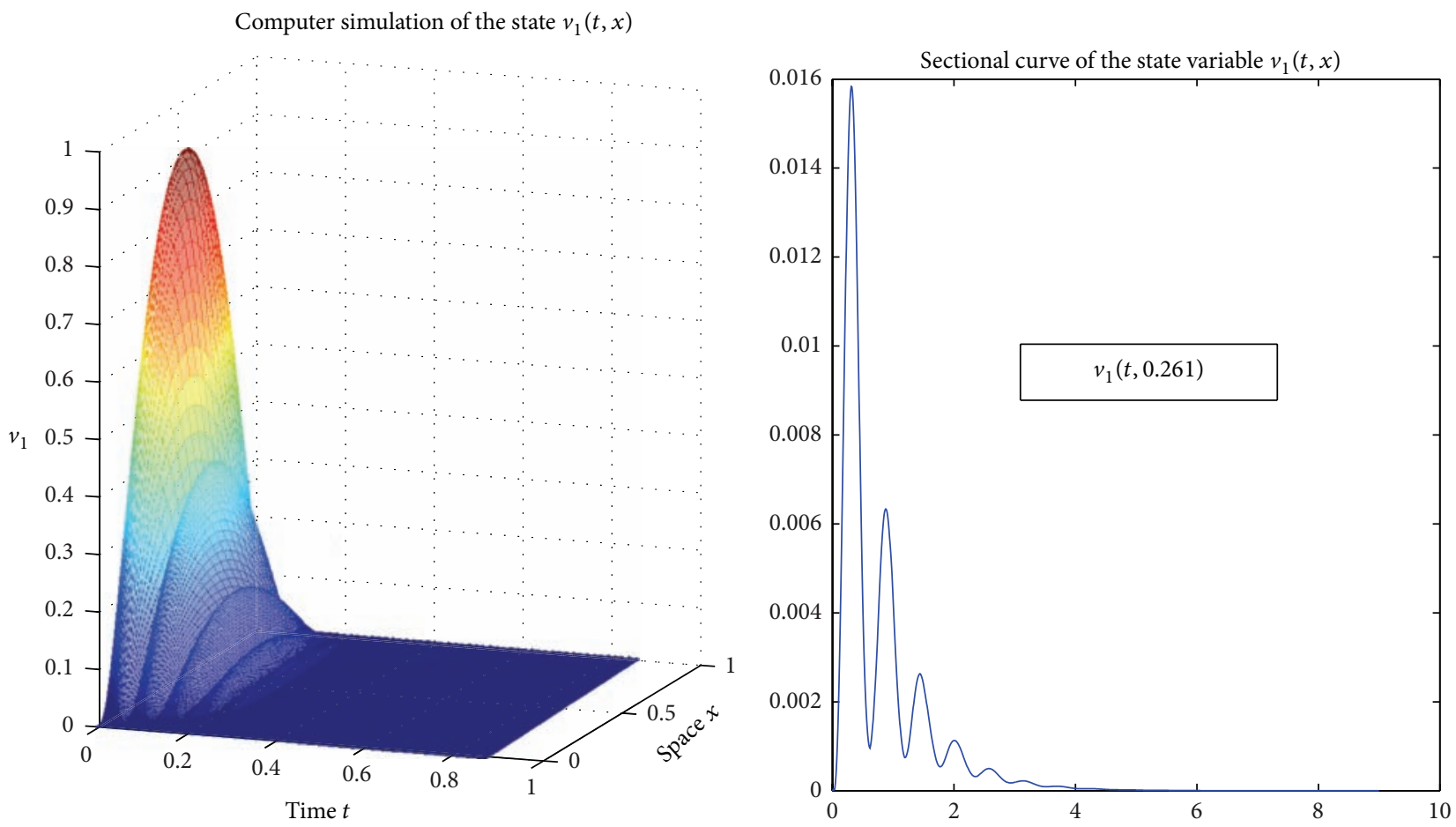

Figure 1: The state variable $v_{1}(t, x)$.

Table 1: Comparisons between [2, Corollary 15] and Theorem 4.

\begin{tabular}{lcccc}
\hline & {$[2$, Corollary 15] } & $\lambda=1$ & $\lambda=10$ & Theorem 4 \\
\hline$t_{\text {min }}$ & $>0$ & $>001$ & $>0$ & $<0$ \\
Feasibility & Unfeasible & Unfeasible & Unfeasible & Feasible \\
Stability & Unknown & Unknown & Unknown & Global asymptotical stability \\
\hline
\end{tabular}

for $i=1,2$. Thereby, we can conclude from Theorem 4 that the null solution for this fuzzy dynamic equation is globally asymptotically stable.

There are some interesting comparisons between [2, Corollary 15] and our Theorem 4 (see Table 1).

Remark 7. Since we consider the role of the nonlinear $p$ Laplace diffusion item in the stability criterion, Example 2 illustrates that our Theorem 4 can judge what [2, Corollary 15] cannot do.

\section{Conclusions}

In this paper, the global asymptotical stability criterion of the nonlinear $p$-Laplace fuzzy T-S dynamical equations with infinite delay was derived by the way of Lyapunov-Krasovskii functional approach and some variational methods in the Sobolev space $W_{0}^{1, p}(\Omega)$. The $p$-Laplace diffusion item plays its role in our stability criterion while the stability criteria obtained in many previous literatures did not contain the diffusion terms. In fact, when $p=2,2$-Laplace is the linear
Laplace, and there are many papers (see, e.g., [25-29]) in which the Laplace diffusion item plays its role in their stability criteria, for the linear Laplace PDEs can be considered in the special Hilbert space $H^{1}(\Omega)$ that can be orthogonally decomposed into the direct sum of infinitely many eigenfunction spaces. However, the nonlinear $p$-Laplace $(p>1$, $p \neq 2$ ) brings great difficulties, for the nonlinear $p$-Laplace PDEs should be considered in the frame of Sobolev space $W^{1, p}(\Omega)$ that is only a reflexive Banach space. Indeed, owing to the great difficulties, the authors only provide in [4] the stability criterion in which the nonlinear $p$-Laplace items play roles in the case of $1<p<2$. Now in this paper, we present the stability criterion in which the nonlinear $p$ Laplace items play roles in the case of $p>2$ or $p>$ 1. Moreover, numerical example shows the effectiveness of the proposed methods. Since the non-linear $p$-Laplace dynamical equations have many physics and engineering background, including the famous Cohen-Grossberg neural networks, a further profound study is very interesting in mathematical theories, methods, and even practice. Up to now, we do not know how the $p$-Laplace $(p>1)$ diffusion 

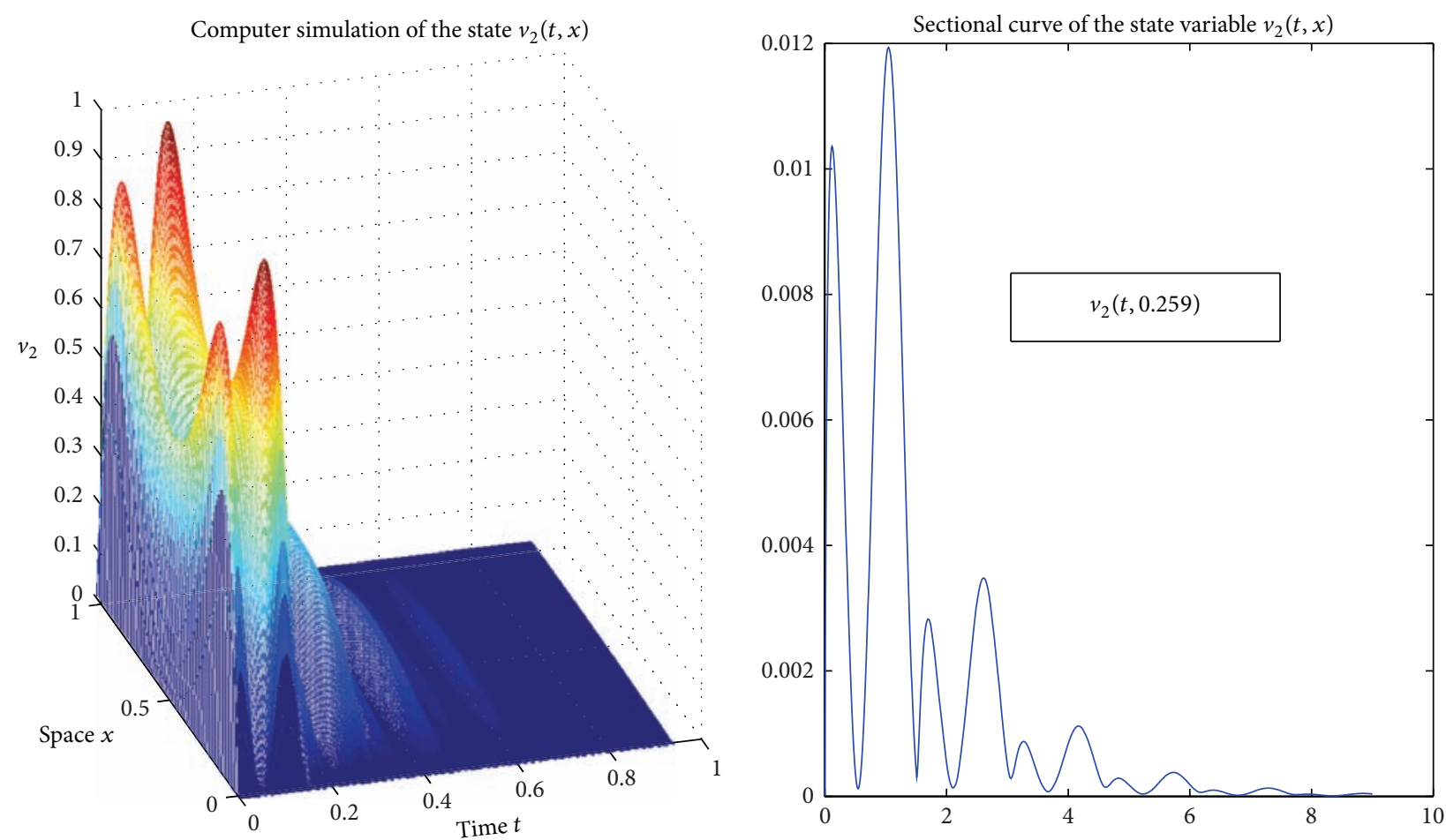

FIGURE 2: The state variable $v_{2}(t, x)$.

item plays a role in $p$-stability criteria. This problem remains open and challenging.

\section{Acknowledgments}

The authors would like to thank the referees for their valuable suggestions. This work was supported by the National Basic Research Program of China (2010CB732501), by the Scientific Research Fund of Science Technology Department of Sichuan Province (2010JY0057, 2012JYZ010), and by the Scientific Research Fund of Sichuan Provincial Education Department (12ZB349, 08ZB002).

\section{References}

[1] Q. F. Pan, Z. F. Zhang, and J. C. Huang, "Stability of the stochastic reaction-diffusion neural network with time-varying delays and p-Laplacian," Journal of Applied Mathematics, vol. 2012, Article ID 405939, 10 pages, 2012.

[2] R. F. Rao, X. R. Wang, S. M. Zhong, and Z. L. Pu, "LMI approach to exponential stability and almost sure exponential stability for stochastic fuzzy Markovian-jumping Cohen-Grossberg neural networks with nonlinear $p$-Laplace diffusion," Journal of Applied Mathematics, vol. 2013, Article ID 396903, 21 pages, 2013.

[3] R. F. Rao and Z. Pu, "Stability analysis for impulsive stochastic fuzzy $p$-Laplace dynamic equations under Neumann or Dirichlet boundary condition," Boundary Value Problems, vol. 2013, article 133, 2013.

[4] R. F. Rao, S. M. Zhong, and X. R. Wang, "Stochastic stability criteria with LMI conditions for Markovian jumping impulsive
BAM neural networks with mode-dependent time-varying delays and nonlinear reaction-diffusion," Communications in Nonlinear Science and Numerical Simulation, vol. 19, no. 1, pp. 258-273, 2014.

[5] R. F. Rao, S. M. Zhong, and X. R. Wang, "Delay-dependent exponential stability for Marko-vian jumping stochastic CohenGrossberg neural networks with p-Laplace diffusion and partially known transition rates via a differential inequality," Advances in Difference Equations, vol. 2013, article 183, 2013.

[6] X. R. Wang, R. F. Rao, and S. M. Zhong, "LMI approach to stability analysis of Cohen-Grossberg neural networks with $p$ Laplace diffusion," Journal of Applied Mathematics, vol. 2012, Article ID 523812, 12 pages, 2012.

[7] X. Zhang, S. Wu, and K. Li, "Delay-dependent exponential stability for impulsive Cohen-Grossberg neural networks with time-varying delays and reaction-diffusion terms," Communications in Nonlinear Science and Numerical Simulation, vol. 16, no. 3, pp. 1524-1532, 2011.

[8] X. Liang and L. S. Wang, "Exponential stability for a class of stochastic reaction-diffusion Hopfield neural networks with delays," Journal of Applied Mathematics, vol. 2012, Article ID 693163, 12 pages, 2012.

[9] Y. T. Zhang, "Asymptotic stability of impulsive reactiondiffusion cellular neural networks with time-varying delays," Journal of Applied Mathematics, vol. 2012, Article ID 501891, 17 pages, 2012.

[10] A. Salem, "Invariant regions and global existence of solutions for reaction-diffusion systems with a tridiagonal matrix of diffusion coefficients and nonhomogeneous boundary conditions," Journal of Applied Mathematics, vol. 2007, Article ID 12375, 15 pages, 2007.

[11] D. J. Higham and T. Sardar, "Existence and stability of fixed points for a discretised nonlinear reaction-diffusion equation 
with delay," Applied Numerical Mathematics, vol. 18, no. 1-3, pp. 155-173, 1995.

[12] V. K. Baranwal, R. K. Pandey, M. P. Tripathi, and O. P. Singh, "An analytic algorithm for time fractional nonlinear reactiondiffusion equation based on a new iterative method," Communications in Nonlinear Science and Numerical Simulation, vol. 17, no. 10, pp. 3906-3921, 2012.

[13] G. Meral and M. Tezer-Sezgin, "The comparison between the DRBEM and DQM solution of nonlinear reaction-diffusion equation," Communications in Nonlinear Science and Numerical Simulation, vol. 16, no. 10, pp. 3990-4005, 2011.

[14] K. Tanaka and M. Sugeno, "Stability analysis and design of fuzzy control systems," Fuzzy Sets and Systems, vol. 45, no. 2, pp. 135156, 1992.

[15] T. Takagi and M. Sugeno, "Fuzzy identification of systems and its applications to modeling and control," IEEE Transactions on Systems, Man, and Cybernetics, vol. 15, pp. 116-132, 1985.

[16] R. F. Rao and Z. Pu, "LMI-based stability criterion of impulsive T-S fuzzy dynamic equations via fixed point theory," Abstract and Applied Analysis, vol. 2013, Article ID 261353, 9 pages, 2013.

[17] Q. K. Song and J. D. Cao, "Global dissipativity on uncertain discrete-time neural networks with time-varying delays," Discrete Dynamics in Nature and Society, vol. 2010, Article ID 810408, 19 pages, 2010.

[18] Q. K. Song, Z. D. Wang, and J. L. Liang, "Analysis on passivity and passification of T-S fuzzy systems with time-varying delays," Journal of Intelligent \& Fuzzy Systems, vol. 24, no. 1, pp. 21-30, 2013.

[19] Q. K. Song, "Stochastic dissipativity analysis on discrete-time neural networks with time-varying delays," Neurocomputing, vol. 74, no. 5, pp. 838-845, 2011.

[20] Q. K. Song and Z. D. Wang, "New results on passivity analysis of uncertain neural networks with time-varying delays," International Journal of Computer Mathematics, vol. 87, no. 1-3, pp. 668-678, 2010.

[21] Q. K. Song and J. Y. Zhang, "Novel results on mesh stability for a class of vehicle following system with time delays," in Advances in Neural Networks-ISNN 2012, vol. 7367 of Lecture Notes in Computer Science, pp. 336-342, 2012.

[22] R. F. Rao and X. R. Wang, "Infinitely many solutions for the resonant quasi-linear equation without Landesman-Lazer conditions," Acta Mathematica Scientia A, vol. 32, no. 4, pp. 744752, 2012.

[23] P. Drábek and R. Manásevich, "On the closed solution to some nonhomogeneous eigenvalue problems with $p$-Laplacian," Differential and Integral Equations, vol. 12, no. 6, pp. 773-788, 1999.

[24] P. Lindqvise, "On the equation $\operatorname{div}\left(|\nabla u|^{p-2} \nabla u\right)+\lambda|u|^{p-2} u=0$," Proceedings of the American Mathematical Society, vol. 109, pp. 159-164, 1990.

[25] J. Pan and S. M. Zhong, "Dynamic analysis of stochastic reaction-diffusion Cohen-Grossberg neural networks with delays," Advances in Difference Equations, vol. 2009, Article ID 410823, 18 pages, 2009.

[26] C. H. Zhou, H. Y. Zhang, H. B. Zhang, and C. Y. Dang, "Global exponential stability of impulsive fuzzy Cohen-Grossberg neural networks with mixed delays and reaction-diffusion terms," Neurocomputing, vol. 91, pp. 67-76, 2012.

[27] C. H. Wang, Y. G. Kao, and G. W. Yang, "Exponential stability of impulsive stochastic fuzzy reaction-diffusion Cohen-Grossberg neural networks with mixed delays," Neurocomputing, vol. 89, pp. 55-63, 2012.
[28] J. Pan and S. M. Zhong, "Dynamical behaviors of impulsive reaction-diffusion Cohen-Grossberg neural network with delays," Neurocomputing, vol. 73, pp. 1344-1351, 2010.

[29] J. Pan, X. Z. Liu, and S. M. Zhong, "Stability criteria for impulsive reaction-diffusion Cohen-Grossberg neural networks with time-varying delays," Mathematical and Computer Modelling, vol. 51, no. 9-10, pp. 1037-1050, 2010. 


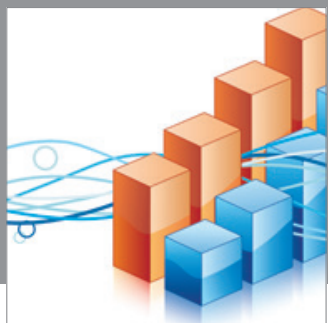

Advances in

Operations Research

mansans

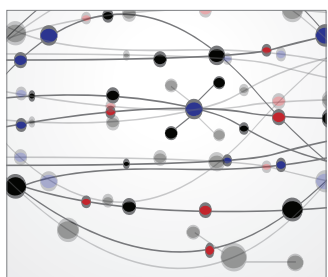

The Scientific World Journal
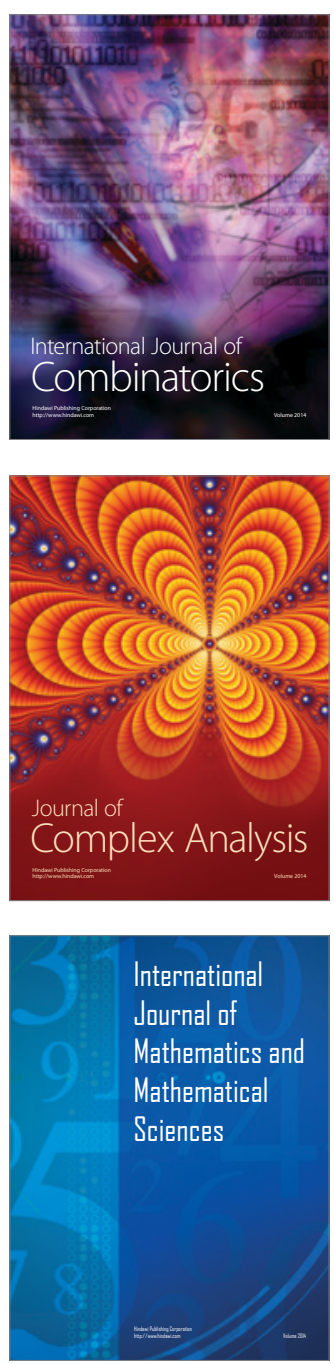
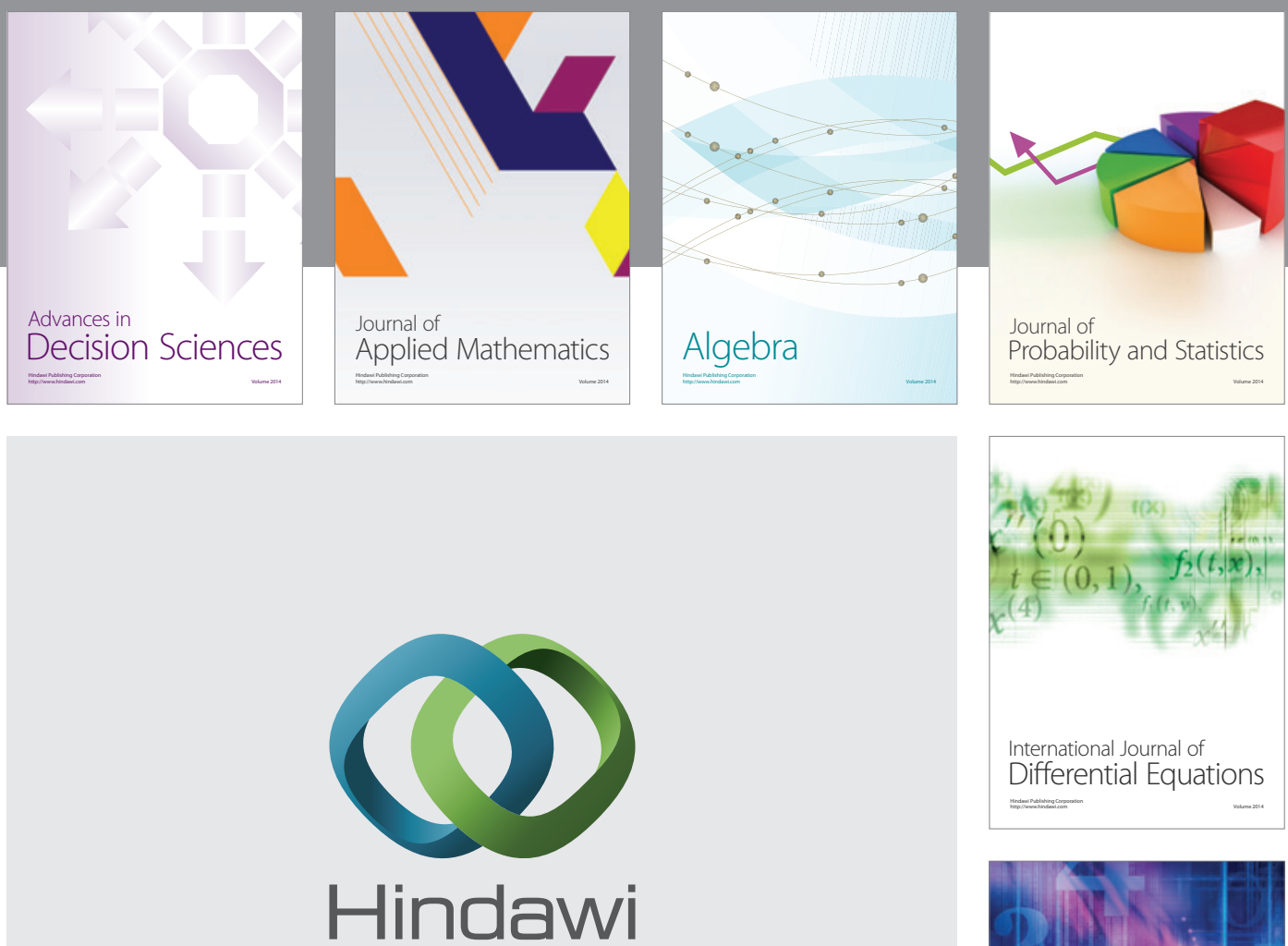

Submit your manuscripts at http://www.hindawi.com
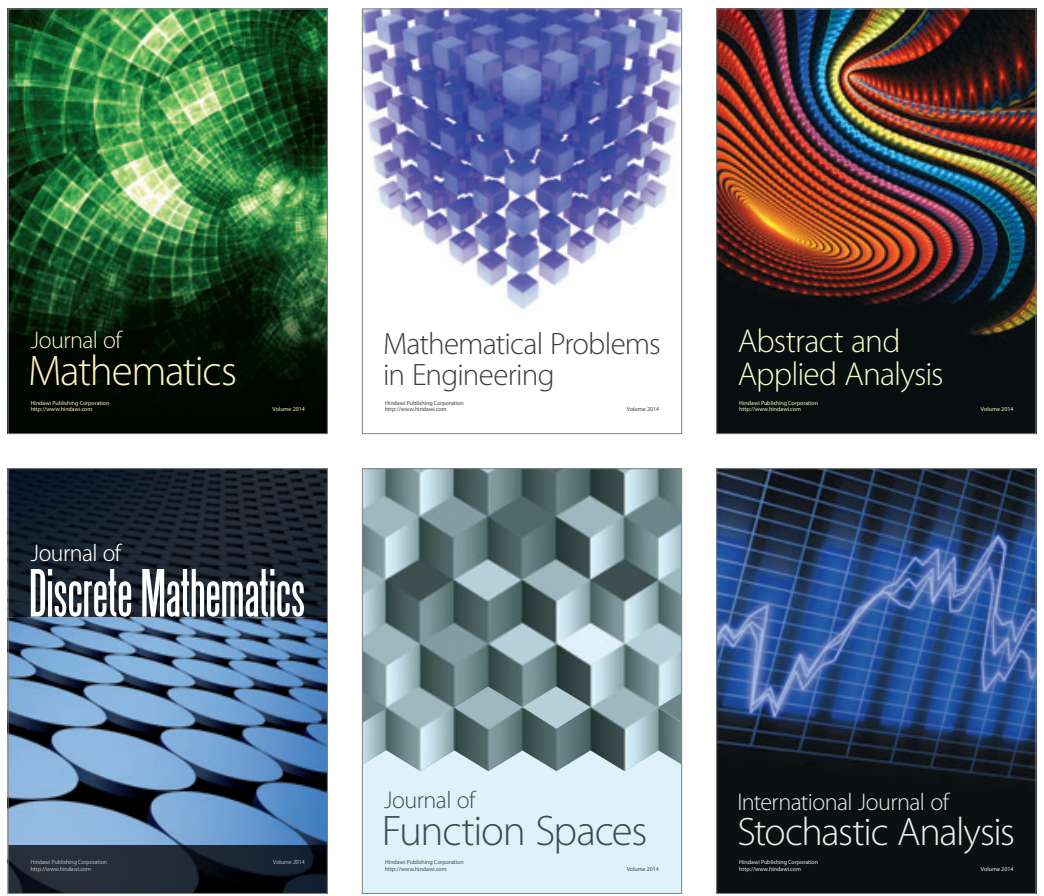

Journal of

Function Spaces

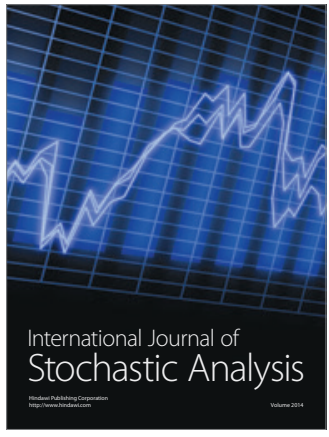

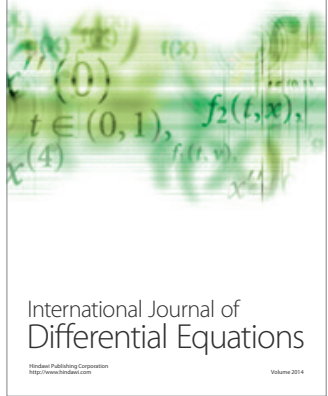
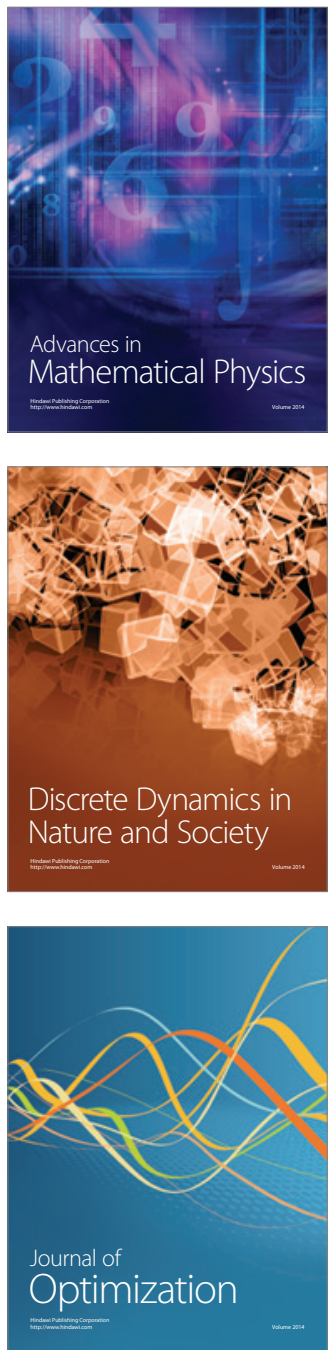